\title{
Laser power stabilization for second-generation gravitational wave detectors
}

\author{
Frank Seifert, Patrick Kwee, Michèle Heurs, Benno Willke, and Karsten Danzmann \\ Max-Planck-Institut für Gravitationsphysik (Albert-Einstein-Institut) and Universität Hannover, Callinstrasse 38, \\ 30167 Hannover, Germany
}

Received March 1, 2006; accepted March 13, 2006; posted April 20, 2006 (Doc. ID 68575)

\begin{abstract}
We present results on the power stabilization of a Nd:YAG laser in the frequency band from $1 \mathrm{~Hz}$ to $100 \mathrm{kHz}$. High-power, low-noise photodetectors are used in a dc-coupled control loop to achieve relative power fluctuations down to $5 \times 10^{-9} \mathrm{~Hz}^{-1 / 2}$ at $10 \mathrm{~Hz}$ and $3.5 \times 10^{-9} \mathrm{~Hz}^{-1 / 2}$ up to several $\mathrm{kHz}$, which is very close to the shot-noise limit for $80 \mathrm{~mA}$ of detected photocurrent on each detector. We investigated and eliminated several noise sources such as ground loops and beam pointing. The achieved stability level is close to the requirements for the Advanced LIGO gravitational wave detector. (C) 2006 Optical Society of America OCIS codes: $040.5160,140.3570,270.2500$.
\end{abstract}

Laser diode pumped solid-state laser systems are exquisitely suited for precision measurement, e.g., as the light sources for laser interferometric gravitational wave detectors such as GEO600 (Ref. 1) and LIGO. ${ }^{2}$ To achieve the sensitivity goals for the second generation of laser interferometric gravitational wave detectors such as Advanced LIGO ${ }^{3}$ one must take care to eliminate noise sources, such as laser amplitude noise, which may limit sensitivity. Current plans for the Advanced LIGO detector call for the use of a $180 \mathrm{~W}$ laser system. Increased laser power will result in increased radiation pressure on the suspended mirrors, and fluctuations in the laser power will thus cause fluctuations in the mirror positions and result in additional noise in the gravitational wave signal channel. The most demanding laser amplitude noise requirement for the Advanced LIGO detector is $2 \times 10^{-9} \mathrm{~Hz}^{-1 / 2}$ at $10 \mathrm{~Hz}$.

Several previous laser power stabilization experiments $^{4-7}$ produced residual noise levels of $10^{-8} \mathrm{~Hz}^{-1 / 2}$ or less for frequencies above $10 \mathrm{~Hz}$ up to several $\mathrm{kHz}$. Those papers showed that power stabilization is exceedingly difficult at low frequencies (below a few hundred Hz). All measurements are limited by an unknown source of excess noise. For higher frequencies the experiments are almost shot-noise or loop-gain limited. In this Letter we demonstrate what is to our knowledge the best stability level achieved so far in the frequency range from $10 \mathrm{~Hz}$ to $10 \mathrm{kHz}$. We present a detailed analysis of the noise sources and set limits on fluctuations in auxiliary degrees of freedom and coupling constants.

The experimental layout used in this work is shown in Fig. 1. The laser was an Innolight Mephisto nonplanar ring oscillator with a total output power of $800 \mathrm{~mW}$ at $1064 \mathrm{~nm}$ and an internal noise eater to reduce the relative intensity noise at the relaxation oscillation frequency (at $\sim 500 \mathrm{kHz}$ ). The laser was locked by the Pound-Drever-Hall technique to a small Fabry-Perot ring cavity (free spectral range: $714 \mathrm{MHz}$; finesse $=4100$ ), the so-called pre-mode cleaner (PMC), ${ }^{8}$ which acts as a spatial mode filter that reduces laser beam geometry fluctuations and beam pointing. This is important because the respon- sitivity of the photodiodes varies across their surfaces, ${ }^{9}$ and variations in the beam position on the photodiodes lead to variations in the photocurrent. A multiaxis piezoelectric mirror placed at the output of the PMC can be used to investigate the coupling between beam pointing and apparent power fluctuations on the photodiodes.

At the output of the PMC the light is split into four beams of identical power, which are aligned with similar optical path lengths onto photodetectors. A quadrant photodetector (Centronic QD50) is used to measure the pointing of the laser beam at the output of the PMC. For intensity noise detection, thermoelectrically cooled $2 \mathrm{~mm}$ InGaAs photodiodes from Hamamatsu ${ }^{10}$ are used in a special transimpedance configuration $\left(R_{\text {TIA }}=100 \Omega\right)$, which enables both lownoise and high-current operation. A reverse bias of $8 \mathrm{~V}$ was chosen to trade off the heat dissipation versus linear operating range. In this configuration the complete photodetector showed a constant slope effi-

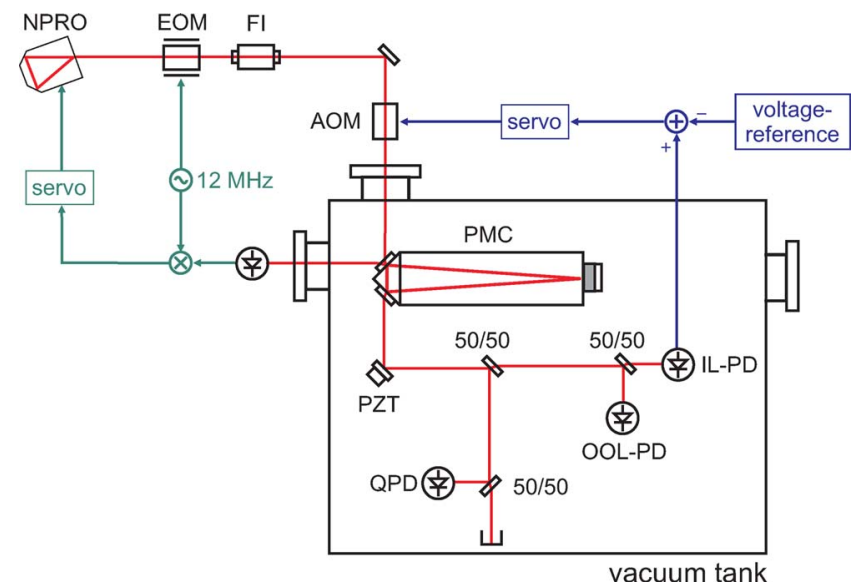

Fig. 1. (Color online) Experimental setup: The laser (NPRO) is locked to a ring cavity (PMC). The control signal for the power stabilization comes from the in-loop detector (IL-PD), and the out-of-loop detector (OOL-PD) is used as an independent sensor. An acousto-optical modulator $(\mathrm{AOM})$ is used as the power actuator. A quadrant photodiode (QPD) is used to measure pointing of the laser beam at the output of the PMC. EOM, electro-optical modulator; FI, Faraday isolator; PZT, piezoelectrically controlled mirror. 


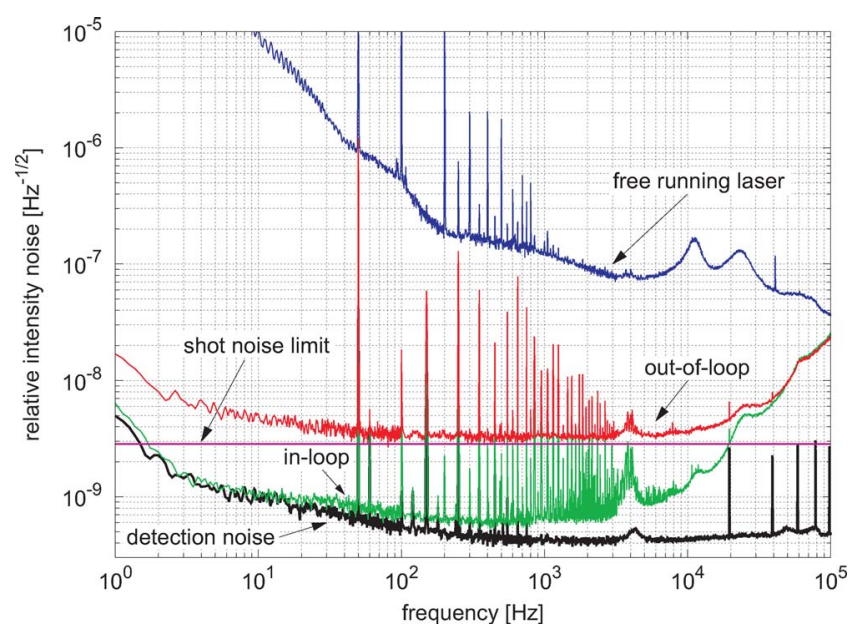

Fig. 2. (Color online) Performance of the power stabilization loop: Relative intensity noise as measured by in-loop and out-of-loop photodetectors. The measurements were taken with $80 \mathrm{~mA}$ of photocurrent. Also shown: freerunning laser noise, detection noise (photodetector dark noise, amplifier and analyzer noise), and the calculated shot-noise limit for this measurement.

ciency up to $150 \mathrm{~mW}$ of optical power. For this experiment the light level incident onto the photodetectors was approximately $110 \mathrm{~mW}$, with a measured quantum efficiency of $\approx 86 \%$, and was limited by the longterm cooling capability of the built-in thermoelectric cooler.

For power stabilization two identical photodetectors are used, one for the stabilization itself (in-loop detector) and the other for an independent measurement (out-of-loop detector). The in-loop photodetector signal was compared with an ultrastable secondorder low-pass filtered voltage reference (AD587, ${ }^{11}$ filter corner frequency $0.048 \mathrm{~Hz}$ ) and was stabilized via feedback to an acoustic-optic modulator at the input of the PMC. The control loop is dc-coupled. An acousto-optic modulator was chosen to provide an actuator that is independent of the laser system used. Hence our setup can be used for many different lasers. Furthermore, a coupling of the feedback signal into other laser parameters (e.g., frequency or pointing) can be avoided.

The control servo was designed with an upper unity gain point at around $100 \mathrm{kHz}$ to achieve high gain at low frequencies. The pole of the cavity is at $86 \mathrm{kHz}$, which has to be taken into account in the design of the control loop. The gain at frequencies below $100 \mathrm{~Hz}$ was at least $100 \mathrm{~dB}$, which in the absence of additional noise sources should have been sufficient to reduce the technical laser amplitude fluctuations to the shot noise level in the sensing photodetectors or to the noise level of the voltage reference used.

The complete detection system (including the optical resonator) is placed in a vacuum tank to minimize the influence of air currents. All experiments were performed at low vacuum (pressure, $<1 \mathrm{hPa}$ ).

The performance of the system is shown in Fig. 2 . The detection noise is dominated by the combined dark noise of the photodetectors and the fast Fourier transform analyzer (Stanford Research Model SRT785 with a homemade battery powered preamp- lifier). The dark noise is comfortably far below the measured out-of-loop intensity noise level. The shot noise for each photodetector $\left(i_{\mathrm{ph}}=80 \mathrm{~mA}\right)$ is 2 $\times 10^{-9} \mathrm{~Hz}^{-1 / 2}$. For low frequencies the in-loop measurement is limited by the noise level of the voltage reference (comparable with the detection noise level); for frequencies higher than $3 \mathrm{kHz}$, by the loop gain.

The out-of-loop measurement is fundamentally limited by the uncorrelated sum of the shot noise on the in-loop and out-of-loop detectors. Summing the shot noise from both photodetectors in quadrature gives a minimum out-of-loop measurement noise of $2.8 \times 10^{-9} \mathrm{~Hz}^{-1 / 2}$. In addition, the noise of the voltage reference used to set the operation point of the control loop is imprinted on the light. The out-of-loop measurement of $3.5 \times 10^{-9} \mathrm{~Hz}^{-1 / 2}$ for $\approx 100 \mathrm{~Hz}$ up to a few $\mathrm{kHz}$ indicates that an additional technical noise source of the same size as the shot noise contaminates either the in-loop measurement and is imprinted on the light by the control loop or leads to excess noise in the out-of-loop measurement. Most probably a combination of both effects describes the measurement best. For Fourier frequencies below $100 \mathrm{~Hz}$ the residual out-of-loop noise was higher. At the lowest frequency of concern for ground-based gravitational wave detectors such as the Advanced LIGO detector $(10 \mathrm{~Hz})$ the noise increased to 5 $\times 10^{-9} \mathrm{~Hz}^{-1 / 2}$.

Various electronic noise sources were characterized, and it could be observed that the residual noise level at low frequencies is highly sensitive to small leakage currents caused by ground loops. Even weak ground loops (e.g., owing to capacitive coupling) lead to higher noise levels, but even when using differential inputs one has to heed floating potentials because of finite common-mode rejection of the input stages. Finally, single-ended signal transmission was chosen, and all electronic subassemblies were powered independently. Cables between subassemblies have been shortened to a minimum to reduce electromagnetic coupling into the system. After careful optimization of electronics and cabling, a rising noise level at lower frequencies was still observable. Slew rate limits of the amplification stages caused by electromagnetic interference were ruled out.

Polarization jitter of the incoming laser beam in combination with polarization-dependent beam splitting could cause differential intensity variations at the in-loop and out-of-loop photodetectors. Further purification of the polarization of the incoming laser beam by means of high extinction polarizing beam splitters at the input of the PMC did not lead to higher stability.

Frequency noise could be converted to intensity noise, e.g., owing to wavelength-dependent response of the photodiodes or the splitting ratio of the power beam splitters. A noise estimation with the freerunning frequency noise of the nonplanar ring oscillator showed that the contribution is much smaller, and different locking arrangements showed no improvement.

To quantify the effect of beam pointing, the spatial uniformity of the implemented photodiodes was mea- 
sured in an additional experiment with a spatial resolution of $10 \mu \mathrm{m}$ and gave a relative sensitivity gradient as large as $2.2 \times 10^{-5} / \mu \mathrm{m} .{ }^{12}$ In combination with a measurement of the beam pointing at the output of the PMC, a projection of the pointing noise coupling into relative intensity noise measurement showed a negligible influence at the current intensity noise level. Note that the beam center had to be aligned to the position on the photodiode with the smallest efficiency gradient to an accuracy of $300 \mu \mathrm{m}$.

Uncorrelated temperature fluctuations of both photodiodes could cause power fluctuations. A typical value for the responsivity temperature coefficient for InGaAs photodiodes is $0.039 \% / \mathrm{K}^{9}$ Therefore it is necessary that a temperature stability of $\approx 5 \mu \mathrm{K}$ is achieved in order not to be limited by temperature fluctuations. Since the photodiodes with built-in thermoelectric coolers used in our experiment contained only one temperature sensor, an out-of-loop measurement of the temperature stability was not possible. However, switching from temperature controlled operation of the photodiode to constant current operation of the thermoelectric cooler yielded the same outof-loop performance of the power stabilization.

Low-frequency noise in the photodiodes itself might become relevant at low frequencies and is in our opinion the prime candidate to explain the excess noise below $100 \mathrm{~Hz}$. Several groups have shown that the noise spectrum of the dark current and the photocurrent depends on several semiconductor parameters and also on operating parameters such as reverse bias voltage, wavelength of the incident light, and illumination level. ${ }^{13-16}$ First experiments with a set of two large-area InGaAs photodiodes in a balanced detection scheme showed a comparable behavior to investigations ${ }^{17}$ of $75 \mu \mathrm{m}$ InGaAs photodiodes under different bias and illumination conditions. For frequencies above a few hundred hertz our experiments showed shot noise-limited performance, and for lower frequencies the noise level increased proportionally to the detected optical power. This result could explain the out-of-loop performance because the detection noise level of the photodetector is measured without light and is therefore not the actual detection limit with a certain amount of optical power on the photodiode.

In conclusion, we have achieved power stabilization of a Nd:YAG laser of $5 \times 10^{-9} \mathrm{~Hz}^{-1 / 2}$ at $10 \mathrm{~Hz}$, which is very close to the requirements for the Advanced LIGO gravitational wave detector. As was already reported by Rollins et al., ${ }^{4}$ electronic grounding noise was discovered to be a critical issue. Nevertheless, at this time it is not known what the limiting factor of the out-of-loop performance is, especially at low frequencies. Further work to identify the limiting noise source at low frequencies will be required. A detailed analysis of low-frequency noise in different commercially available large-area InGaAs photodiodes is currently under investigation.

This research is supported by the Deutsche Forschungsgemeinschaft Sonderforschungbereich 407, "Quantum-Limited Measurement Processes with Atoms, Molecules and Photons" and the Volkswagon Foundation, Germany. F. Seifert's e-mail address is frank.seifert@aei.mpg.de.

\section{References}

1. S. Hild, H. Grote, J. R. Smith, and M. Hewitson, J. Phys.: Conf. Ser. 32, 66 (2006).

2. D. Sigg, Class. Quantum Grav. 21, S409 (2004).

3. A. Weinstein, Class. Quantum Grav. 19, 1575 (2002).

4. J. Rollins, D. Ottaway, M. Zucker, R. Weiss, and R. Abbott, Opt. Lett. 29, 1876 (2004).

5. B. W. Barr, K. A. Strain, and C. J. Killow, Class. Quantum Grav. 22, 4279 (2005).

6. R. Abbott and P. King, Rev. Sci. Instrum. 72, 1346 (2000).

7. F. Nocera, Class. Quantum Grav. 21, S481 (2004).

8. B. Willke, N. Uehara, E. Gustafson, R. Byer, P. King, S. Seel, and R. Savage, Jr., Opt. Lett. 23, 1704 (1998).

9. Perkin-Elmer Optoelectronics, "Large-area InGaAs photodiodes," data sheet, www.perkinelmer.com(2000).

10. Hamamatsu Photonics K.K., "InGaAs PIN photodiode G8605 series," data sheet, www.hamamatsu.com (2001).

11. Analog Devices, "High precision $10 \mathrm{~V}$ reference," data sheet, www.analog.com (2005).

12. P. Kwee, "Charakterisierung von Lasersystemen für Gravitationswellendetektoren," diploma thesis (University of Hannover, 2005).

13. A. M. Joshi, G. H. Olsen, V. S. Ban, E. Mykietyn, M. J. Lange, and D. T. Mohr, IEEE Trans. Electron. Devices 40, 303 (1993).

14. M. Rimini-Döring, A. Hangleiter, S. Winkler, and N. Klötzer, Appl. Phys. A 54, 120 (1992).

15. J. Kimmerle, W. Kuebart, E. Kuehn, O. Hildebrand, K. Loesch, and G. Seitz, in Noise in Physical Systems and 1/f Noise, M. Savelli, G. Lecoy, and J.-P. Nougier, eds. (Elsevier Science, 1983), pp. 249-252.

16. T. G. M. Kleinpenning, J. Vac. Sci. Technol. A 3, 176 (1985).

17. L. He, Y. Lin, A. D. van Rheenen, A. van der Ziel, A. Young, and J. P. van der Ziel, J. Appl. Phys. 68, 5200 (1990). 